\title{
A Prospective, Randomized, Open-Label Study to Evaluate Two Management Strategies for Gastrointestinal Symptoms in Patients Newly on Treatment with Dabigatran
}

Daniel O’Dea $\cdot$ Jacqueline Whetteckey $\cdot$ Naitee Ting

Received: April 27, 2016/Published online: October 5, 2016

(C) The Author(s) 2016. This article is published with open access at Springerlink.com

\section{ABSTRACT}

Introduction: In the pivotal RE-LY trial, dabigatran etexilate (DE) at the dose of 150-mg twice daily (BID), significantly reduced total stroke and ischemic stroke compared with warfarin in patients with non-valvular atrial fibrillation (NVAF), while the 110-mg BID dose had efficacy equivalent to warfarin, and major bleeds were significantly reduced. Both DE regimens were generally well tolerated; however, approximately $4 \%$ of the patients discontinued treatment with DE due to gastrointestinal (GI) discomfort.

Enhanced content To view enhanced content for this article go to http://www.medengine.com/Redeem/ B495F06071D58495.

Electronic supplementary material The online version of this article (doi:10.1007/s40119-016-0071-5) contains supplementary material, which is available to authorized users.

D. O'Dea $(\bowtie)$

Division of Cardiology, Vassar Brothers Medical Center, 1 Columbia Street, Poughkeepsie, NY 12540, USA

e-mail: dodea@Health-quest.org

J. Whetteckey · N. Ting

Boehringer Ingelheim Pharmaceuticals, Inc., 900

Ridgebury Road, Ridgefield, CT 06877, USA
Methods: Clinical trial NCT01493557 was a multicenter, randomized, active control, open-label study to assess the efficacy of two simple GI symptom (GIS) management strategies in DE-treated patients who developed GIS: (1) concurrent treatment with the proton pump inhibitor pantoprazole (DE-P), or (2) ingestion of DE after a meal (DE-M). Patients were initially randomized to either GIS management strategy. If the first did not resolve their GIS, patients had the option to "add on" the alternative strategy.

Results: A total of 1067 patients with NVAF received DE therapy BID for 3 months (United States, $150-\mathrm{mg}$ or $75-\mathrm{mg}$; Canada, $150-\mathrm{mg}$ or 110-mg). Of these, 117 (11\%) patients reported GIS and were randomized to one of two GIS management strategies. At 4 weeks, a significantly higher rate of complete or partial effectiveness was observed in patients on DE-P than in those receiving DE-M, [50/58 (86.2\%) versus $40 / 59$ (67.8\%), respectively; $p=0.0273$ ]. Patients with ongoing GIS were asked to "add on" the alternate strategy for an additional 4 weeks. Overall, 92/117 (78.6\%) of randomized patients experienced complete or partial effectiveness using either the initial 
strategy or a combination of the two strategies: DE-P, 47 (81.0\%); and DE-M, 45 (76.3\%, no significant difference) (by initial strategy).

Conclusion: The majority of patients enrolled either did not experience GIS at all, or their GIS resolved using either one individually, or a combination of the two strategies described.

Trial registration: http://www.ClinicalTrials. gov identifier: NCT01493557.

Keywords: Dabigatran; Dyspepsia;

Gastrointestinal symptoms; Pantoprazole

\section{INTRODUCTION}

Dabigatran [dabigatran etexilate (DE) mesylate], a direct-acting oral anticoagulant (DOAC), is a prodrug that is rapidly converted by a serum esterase to active dabigatran, a potent, direct, competitive inhibitor of thrombin [1]. DE is approved in many countries around the world for the reduction in risk of stroke and systemic embolism in non-valvular atrial fibrillation (NVAF) and a number of other indications. In the United States, DE is also approved for the treatment of deep vein thrombosis (DVT) and pulmonary embolism (PE), the reduction in risk of DVT and PE in patients who have been previously treated, and the prophylaxis of DVT and PE in patients who have undergone hip replacement surgery [2]. In Canada, DE is approved for the prevention of venous thromboembolism (VTE) in patients who have undergone elective total hip or total knee replacement surgery, for the treatment of VTE and the prevention of recurrent DVT and $\mathrm{PE}$ and, in patients with atrial fibrillation (AF) when anticoagulation is appropriate, the prevention of stroke and systemic embolism [3].

The pivotal global Randomized Evaluation of Long-term Anticoagulation Therapy (RE-LY) trial (http://www.ClinicalTrials.gov identifier:
NCT00262600), compared DE [110-mg and 150-mg twice daily (BID)] with adjusted-dose warfarin for a median follow-up period of 2 years in 18,113 patients with NVAF at risk for stroke [4]. For the primary outcome of stroke or systemic embolism, DE 110-mg was non-inferior to warfarin, while the 150-mg dose of DE was superior to warfarin [relative risk (RR) 0.65 ; 95\% confidence interval (CI) $0.52-0.81 ; p<0.001$ for superiority] [5]. In the RE-LY trial, the two DE regimens were generally well tolerated. However, dyspepsia-like gastrointestinal (GI) symptoms (GIS) (defined as upper abdominal pain, abdominal pain, abdominal discomfort, and dyspepsia) were reported more often in patients receiving DE (110-mg: 11.8\%; 150-mg: $11.3 \%)$ than in those receiving warfarin (5.8\%) [4]. Post hoc analysis of the RE-LY database showed that $4 \%$ of patients stopped taking dabigatran because of non-bleeding upper GI adverse events (AEs), mostly within 3 months of initiating DE therapy [6].

The long-term follow-up to RE-LY, the RELY-ABLE study (http://www.ClinicalTrials. gov identifier: NCT00808067), followed 5851 patients (48.9\%) who continued to receive their blinded DE dose (110-mg or 150-mg BID) according to the original RE-LY study design [7]. Of the patients who experienced dyspeptic symptoms in RE-LY, the proportion who entered the RELY-ABLE study was only slightly smaller (45\%). The baseline characteristics of this subgroup were generally similar to that of the original study population receiving DE in RE-LY [7]. In response to a questionnaire, these patients self-reported a number of strategies to address their dyspepsia, with the largest number of patients reporting symptom relief by either co-administering a proton pump inhibitor or by taking DE with a meal [7].

However, the optimal strategy to prevent or treat DE-associated GIS had not been formally tested in a randomized trial. Therefore, the 
present clinical trial, performed in the United States and Canada, compared the relative efficacy of two proposed strategies to manage GIS: (1) to add the proton pump inhibitor pantoprazole [40-mg once daily (OD)] in the morning, or (2) to take DE within 30 min after a meal. At the time of initiation of this trial, DE was approved for the reduction in risk of stroke and systemic embolism in patients with NVAF in the United States, and also for prevention of VTE in patients undergoing knee or hip replacement in Canada. However, DE was not yet approved for the prevention of VTE in patients undergoing hip replacement in the United States. Therefore, NVAF was an inclusion criterion.

\section{METHODS}

\section{Patients}

Patients recruited to participate in the present clinical trial were adults (at least 18 years old) with documented NVAF who were not previously treated with DE (or not treated for more than 7 days). Patients were excluded from trial entry if they had protocol-defined GIS (see below) within the 2 weeks prior to enrollment, GI bleeding within the prior year, or any history of symptomatic or endoscopically documented gastroduodenal ulcer or diverticulitis. Patients with nausea and/or vomiting within 2 weeks prior to enrollment could be included if their symptoms were clearly associated with a self-limited acute or febrile illness. If patients had creatinine clearance $<15 \mathrm{ml} / \mathrm{min}$ (sites in the United States) or $<30 \mathrm{ml} / \mathrm{min}$ (sites in Canada), or were on renal replacement therapy (dialysis), then they were not eligible for the trial. In addition, patients were excluded if they had contraindications to $\mathrm{DE}$ or associated excipients, pantoprazole, or other proton pump inhibitors.

\section{Trial Design}

The present trial (http://www.ClinicalTrials.gov identifier: NCT01493557) had a multicenter, randomized, active control open-label design to assess the efficacy of two GIS management strategies in patients treated with DE at multiple sites in the United States and Canada. The trial design was open label, because postprandial administration of DE was not suitable for blinding. The 3-month window was selected since this was the time frame during which the difference in GIS rates between the dabigatran and warfarin cohorts occurred in the RE-LY trial [4]. A 4-week period for assessing GIS management strategies was selected because the GIS categories reported in the RE-LY trial were expected to respond (or not) to treatment within that time frame.

Eligible patients were placed on DE therapy according to the current United States or Canadian label for 3 months; dosing was either $150-\mathrm{mg}$ or $75-\mathrm{mg}$ BID at sites in the United States, and was either 150-mg or 110-mg BID at sites in Canada. Patients who experienced GIS at any time during the 3-month treatment period were directed to report their symptoms. Reportable GIS was defined as: heartburn, epigastric burning, epigastric pain, epigastric discomfort, upper abdominal discomfort, upper abdominal pain, upper abdominal burning, postprandial fullness, upper abdominal bloating, nausea, vomiting, excessive burping/belching, indigestion, or regurgitation of bitter fluid. Lower GIS, including diarrhea, constipation, and lower abdominal pain, was not included as reportable GIS in this trial. Patients who did not report GIS during the 3-month DE 
treatment period were not followed further in this trial.

Patients who reported GIS at any time during the 3-month DE treatment period (GIS-1, via phone call) were clinically assessed at GIS-visit 2 (in person within $72 \mathrm{~h}$ of the initial phone call) and, if symptoms were still ongoing, were to then be randomized according to a $1: 1$ ratio to one of two simple GIS management strategies for 4 weeks, with each serving as an active treatment control arm for the other: (1) DE plus pantoprazole 40-mg OD in the morning; or (2) DE administered within $30 \mathrm{~min}$ after a meal (morning and evening) (Fig. 1). If the patient continued to experience GIS after 4 weeks of the initial GIS management strategy, then the other GIS management strategy was to be "added on" to the initial strategy (according to the schedule for each). To ensure a consistent baseline for assessing responses to the strategy of administering DE within 30 min after a meal, all entered patients were initially instructed to take DE with 8 fluid ounces (approximately $237 \mathrm{ml}$ ) of water, and not within the initial $30 \mathrm{~min}$ following a meal. Strategy randomization was by random code, generated using validated randomization software via a third-party Interactive Voice Response System/Interactive Web Response

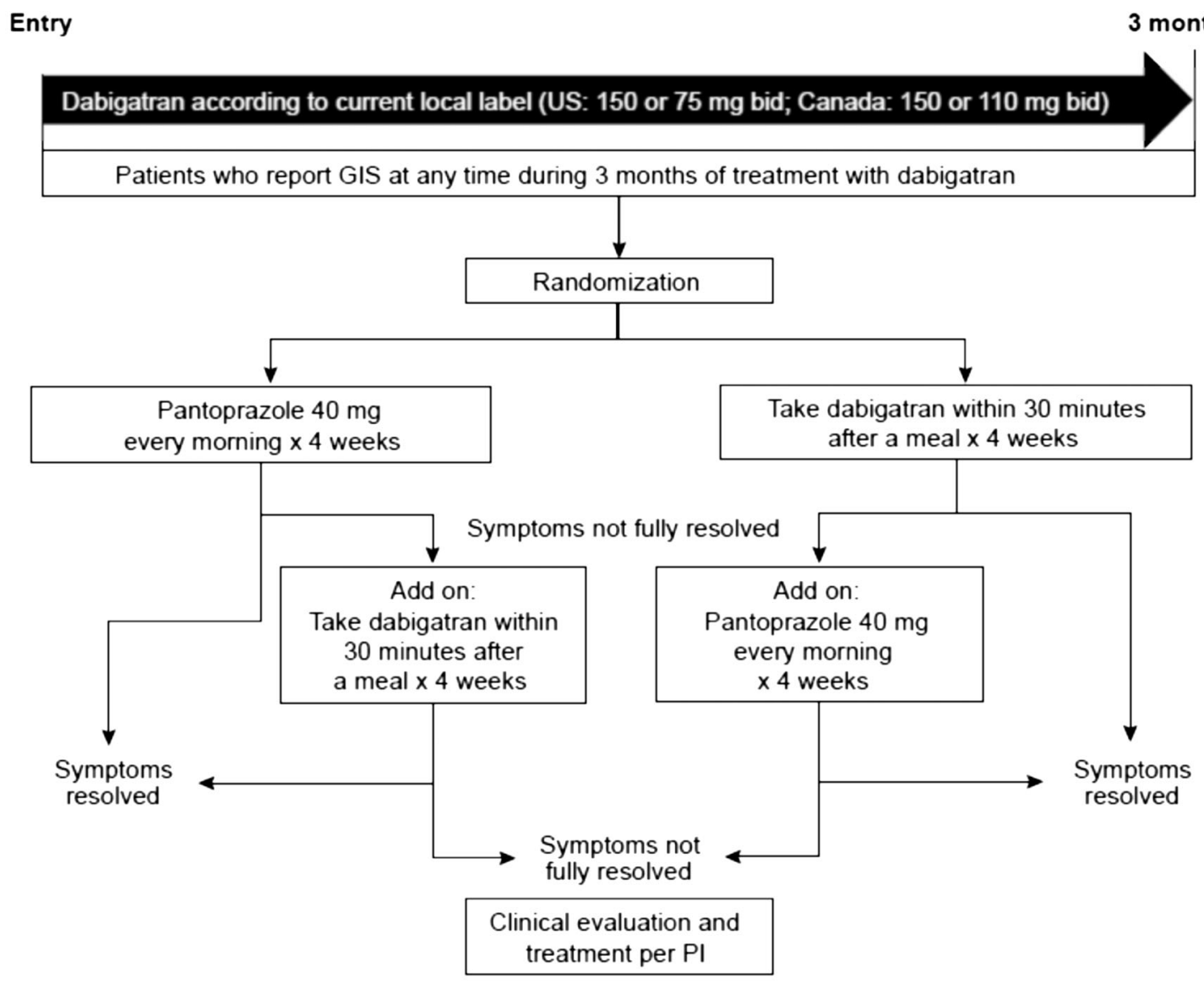

$B I D$, twice daily: $G / S$, gastrointestinal symptom: $P I$, principal investigator

Fig. 1 GIS trial design 
System $\left[\mathrm{XRS}^{\circledR}\right.$ User Requirement Specification (URS) version 6, Almac Clinical Technologies, Souderton PA].

GIS incidence and grade were assessed by each patient by completing the Gastrointestinal Symptoms Rating Scale (GSRS) questionnaire, which included questions regarding the level of discomfort experienced during the previous week as a result of 15 GI conditions. The GSRS questionnaire was completed at each attended face-to-face visit (GIS-2, GIS-4, GIS-6, GIS-8, and GIS-10). Additional visits (GIS-3, GIS-5, GIS-7, and GIS-9) were conducted via telephone calls on alternate weeks.

If a patient continued to experience GIS after 4 weeks on his or her initial management strategy, the alternate GIS management strategy was to be "added on" to the initial strategy. If a patient still continued to have GIS after an additional 4 weeks of management, he or she was to be evaluated and treated for the continued GIS according to the investigator's judgment. Thus, patients who developed and reported GIS were treated with DE during the initial 3-month period, and then up to eight additional weeks, depending on whether symptoms resolved fully with the first management strategy or if they required the "add on" strategy. Patients with GIS were followed up at least weekly until 1 week after the GIS resolved, an underlying diagnosis was identified, or the investigator and sponsor agreed that no further follow-up was necessary. After completion of the trial, further management was at the discretion of the investigator.

Patient compliance with assigned treatment was defined as consumption of $80-120 \%$ of pills prescribed (pills consumed/pills prescribed $\times$ 100). Patients assigned to the strategy of taking DE after a meal were considered compliant if they reported that they took DE within 30 min after a meal "always" or "most of the time."

\section{Endpoints}

The primary efficacy endpoint was the rate of complete effectiveness (i.e., complete relief of GIS) after 4 weeks of pantoprazole 40-mg OD in the morning, or of DE administration within 30 min after a meal as assessed by patient responses to the GSRS questionnaire. Secondary efficacy endpoints at 4 weeks included the rate of complete and partial effectiveness of either GIS management strategy, and at 8 weeks after the onset of symptoms included the rates of complete, partial, and complete or partial relief of GIS at each week.

The incidence and nature of all reported AEs were recorded during the trial, including primary system organ class and preferred term, severity (mild, moderate, or severe), serious AEs, DE-related AEs, and discontinuations due to AEs.

\section{Statistical Analyses}

Based on prior experience, approximately $6-12 \%$ of patients were expected to report GIS in this trial. Therefore, with a sample size of 1200 patients, approximately 72-144 patients would develop GIS within 3 months of DE treatment. Half of the patients (36-72) developing GIS would be treated with pantoprazole, and half (36-72) would take DE after a meal. A sample size of 1200 patients would allow detection of clinically meaningful differences in efficacy between the two management strategies (90\% versus 50\%), with $80 \%$ or $90 \%$ power, and a one-sided alpha of 0.025 . 
Efficacy endpoints were analyzed using point estimates and 95\% CIs for the differences between the two management strategies, with each serving as the active control for the other. Comparisons across all time points (including week 4 and week 8) were based only on the two GIS management strategies, as randomized at symptom onset. CIs were constructed using the Clopper-Pearson method.

Planned analyses were by intent to treat, which includes all patients who reported GIS during the initial 3-month DE treatment period, and who were randomized to GIS management strategies. Efficacy analyses were performed according to the randomization group each patient was assigned to, regardless of the actual GIS management strategy received. At the 4-week assessment, all patients were included based on last observation carried forward analysis. In contrast, safety analyses were performed according to the GIS management strategy each patient actually received, regardless of the initial randomization assignment. Statistical calculations and graphical presentations were prepared using the SAST System version 9.4 (SAS Institute Inc., Cary, North Carolina).

\section{Compliance with ethics guidelines}

All procedures followed were in accordance with the ethical standards of the responsible committee on human experimentation (institutional and national) and with the Helsinki Declaration of 1964, as revised in 2013. The study was also performed in accordance with the ICH Harmonised Tripartite Guideline for Good Clinical Practice (GCP). All patients provided written informed consent in accordance with all local regulatory and legal requirements of each participating country prior to the initiation of any trial-related procedure.

\section{RESULTS}

\section{Patients}

Demographic data for the randomized patients (treated set) were similar in the two GIS management strategy groups (Table 1). Almost two-thirds of the patients were male $(64.1 \%)$, most were white $(94.9 \%)$, and the mean age was 69.3 years (range 41.0-94.0 years).

The study enrolled 1067 patients who were treated with DE. Of these, 950 patients completed the trial, but were not randomized (Fig. 2). These included 854 patients who completed 3 months of DE, with no reported GIS. Another 48 patients reported GIS at visit 1 (GIS-1). However, 32 of these patients had complete resolution of GIS without treatment, and 16 patients discontinued the study before GIS-2, including four patients who reported that their GIS spontaneously resolved. A final group of 48 patients reported GIS only at the end-of-treatment visit (despite instructions to call the study site if they experienced any GIS), so they were not randomized. These patients either experienced complete resolution of their GIS without treatment (26 patients), discontinued the study early (18 patients), or had ongoing GIS (four patients) at the end-of-treatment visit.

Out of the total of 1067 patients treated with dabigatran, there were 117 (11\%) who reported GIS prior to the end-of-treatment visit and were randomized to either DE plus pantoprazole 40-mg OD in the morning $(n=58)$, or DE taken within $30 \mathrm{~min}$ after a meal $(n=59)$, each strategy for 4 weeks. 
Table 1 Demographic data for randomized patients by GIS management strategy (treated set)

\begin{tabular}{|c|c|c|c|}
\hline Characteristic & DE after a meal $(n=59)$ & DE plus pantoprazole $(n=58)$ & Total $(N=117)$ \\
\hline \multicolumn{4}{|l|}{ Gender, $n(\%)$} \\
\hline Male & $37(62.7)$ & $38(65.5)$ & $75(64.1)$ \\
\hline Female & $22(37.3)$ & $20(34.5)$ & $42(35.9)$ \\
\hline \multicolumn{4}{|l|}{ Race, $n(\%)$} \\
\hline American Indian/Alaskan Native & 0 & $1(1.7)$ & $1(0.9)$ \\
\hline Asian & 0 & $1(1.7)$ & $1(0.9)$ \\
\hline Black/African American & $1(1.7)$ & $3(5.2)$ & $4(3.4)$ \\
\hline White & $58(98.3)$ & $53(91.4)$ & $111(94.9)$ \\
\hline \multicolumn{4}{|l|}{ Ethnicity, $n$ (\%) } \\
\hline Not Hispanic/Latino & $57(96.6)$ & $56(96.6)$ & $113(96.6)$ \\
\hline Hispanic/Latino & $2(3.4)$ & $2(3.4)$ & $4(3.4)$ \\
\hline \multicolumn{4}{|l|}{ Age, years } \\
\hline Mean (SD) & $69.1(11.1)$ & $69.6(10.8)$ & $69.3(10.9)$ \\
\hline Median (min, $\max )$ & $70.0(41.0,94.0)$ & $69.5(42.0,93.0)$ & $70.0(41.0,94.0)$ \\
\hline \multicolumn{4}{|l|}{ Height, $\mathrm{cm}$} \\
\hline Mean (SD) & $172.1(11.8)$ & $173.4(9.7)$ & $172.7(10.8)$ \\
\hline Median (min, max) & $173.0(144.0,206.0)$ & $175.0(144.0,190.0)$ & $175.0(144.0,206.0)$ \\
\hline \multicolumn{4}{|l|}{ Weight, kg } \\
\hline Mean (SD) & $98.0(32.3)$ & $97.1(26.2)$ & $97.5(29.3)$ \\
\hline Median (min, max) & $93.8(36.9,200.9)$ & $91.6(46.6,181.0)$ & $93.3(36.9,200.9)$ \\
\hline \multicolumn{4}{|l|}{ Smoking status, $n(\%)$} \\
\hline Never smoked & $25(42.4)$ & $30(51.7)$ & $55(47.0)$ \\
\hline Ex-smoker & $30(50.8)$ & $24(41.4)$ & $54(46.2)$ \\
\hline Currently smokes & $4(6.8)$ & $4(6.9)$ & $8(6.8)$ \\
\hline \multicolumn{4}{|l|}{ Alcohol status, $n(\%)$} \\
\hline Non-drinker & $19(32.2)$ & $26(44.8)$ & $45(38.5)$ \\
\hline Drinks-no interference ${ }^{\mathrm{a}}$ & $40(67.8)$ & $32(55.2)$ & $72(61.5)$ \\
\hline
\end{tabular}

$D E$ dabigatran etexilate, GIS gastrointestinal symptom, max maximum, min minimum, $S D$ standard deviation

${ }^{a}$ No interference with usual activities because of discomfort

Patients who continued to experience GIS added the alternate strategy for a further 4 weeks. Of the 117 patients who were randomized, $26(22.2 \%)$ of them withdrew before the completion of the trial because of the following reasons [AEs, 18; protocol non-compliance, three; consent withdrawn (non-AE related), one; other, four]. 


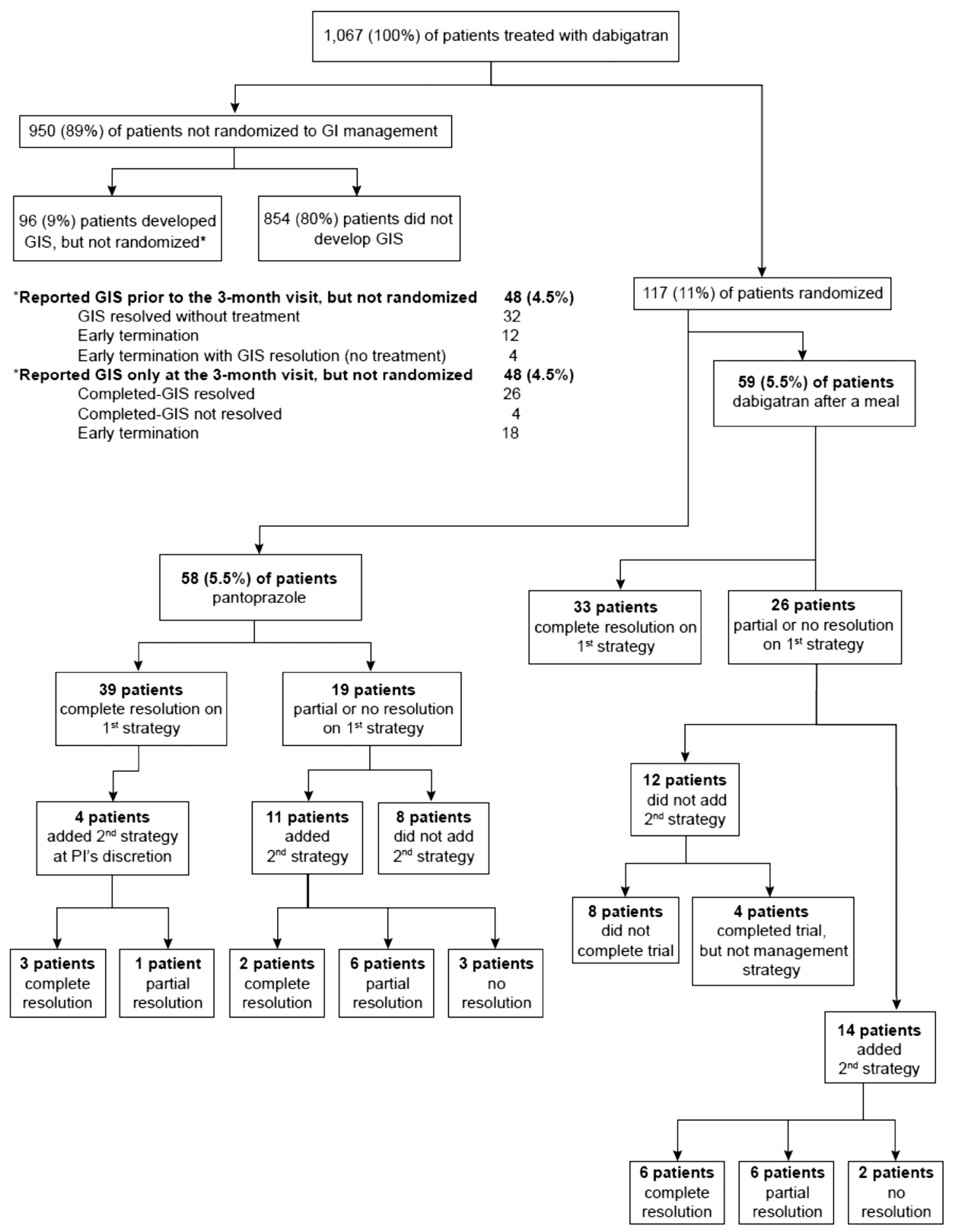

\section{GI, gastrointestinal: $G / S$, gastrointestinal symptom: $P I$, principal investigator}

Fig. 2 Patient outflow 
Table 2 Primary reported GIS at randomization (treated set)

\begin{tabular}{lll}
\hline Event & DE after a meal $(\boldsymbol{n}=\mathbf{5 9 )}$ & DE plus pantoprazole $(\boldsymbol{n}=\mathbf{5 8})$ \\
\hline Heartburn & $27(45.8)$ & $28(48.3)$ \\
Epigastric burning & $2(3.4)$ & $6(10.3)$ \\
Nausea & $3(5.1)$ & $5(8.6)$ \\
Epigastric pain & $5(8.5)$ & $3(5.2)$ \\
Excessive burping/belching & $5(8.5)$ & $3(5.2)$ \\
Upper abdominal discomfort & $5(8.5)$ & $3(5.2)$ \\
Indigestion & $4(6.8)$ & $4(6.9)$ \\
Epigastric discomfort & $3(5.1)$ & 0 \\
Upper abdominal pain & $1(1.7)$ & $2(3.4)$ \\
Regurgitation of bitter fluid & $2(3.4)$ & 0 \\
Upper abdominal bloating & 0 & $2(3.4)$ \\
Upper abdominal burning & $1(1.7)$ & $1(1.7)$ \\
Vomiting & $1(1.7)$ & $1(1.7)$ \\
\hline
\end{tabular}

$D E$ dabigatran etexilate, $G I S$ gastrointestinal symptom

Individual rates for primary GIS at randomization are presented in Table 2 . The most common primary GIS was heartburn, reported by almost half of the patients in each GIS management strategy group.

\section{Efficacy}

The overall effectiveness of using either one or both (as "add on") of the GIS management strategies in this trial is shown in Fig. 2.

At the 4-week assessment, of the 117 patients randomized to either of the GIS management strategies, no significant difference (NSD) was seen in the rates of complete effectiveness reported with either initial strategy: 39/58 $(67.2 \%)$ of patients taking DE plus pantoprazole versus 33/59 (55.9\%) of patients taking DE after a meal $(p=0.2554)$. Partial effectiveness was reported by an additional 11 (19.0\%) patients taking DE plus pantoprazole and 7 (11.9\%) patients taking DE after a meal $(p=0.3165)$. However, when these two outcomes were combined, the rates of complete and partial effectiveness at the 4-week assessment were significantly different, with the DE plus pantoprazole strategy being more effective than the DE after a meal strategy (86.2\% versus $67.8 \%$, respectively; $p=0.0273$; Table 3).

Of the 39 patients in the DE plus pantoprazole group who experienced complete effectiveness at GIS-visit 6 (after 4 weeks of initial management strategy), four patients were allowed to add the second management strategy at the investigator's discretion. For these four patients, only the outcome at GIS-visit 10 (after 4 weeks of the combined GIS management strategies) is included (three patients, complete effectiveness; one patient, partial effectiveness) (Fig. 2).

Of the patients who initiated on the DE plus pantoprazole strategy, 19 reported partial or no resolution. Of these, 11 patients added the 
Table 3 Rates of complete or partial effectiveness at week 4 (LOCF)

\begin{tabular}{|c|c|c|}
\hline & DE after a meal & Pantoprazole \\
\hline Number of patients $[n(\%)]$ & $59(100.0)$ & $58(100.0)$ \\
\hline Number (\%) with complete or partial effectiveness ${ }^{a}$ & $40(67.8)$ & $50(86.2)$ \\
\hline $95 \% \mathrm{CI}(\%)^{\mathrm{b}}$ & $(54.4,79.4)$ & $(74.6,93.9)$ \\
\hline \multicolumn{3}{|l|}{ Comparison versus DE after a meal } \\
\hline Estimate & 18.41 & \\
\hline $95 \% \mathrm{CI}(\%)^{\mathrm{b}}$ & $(0.71,35.98)$ & \\
\hline$p$ value $^{\mathrm{c}}$ & 0.0273 & \\
\hline \multicolumn{3}{|c|}{$\begin{array}{l}C I \text { confidence interval, } D E \text { dabigatran etexilate, GIS gastrointestinal symptom, } L O C F \text { last observation carried forward } \\
\text { a Complete effectiveness is defined as all primary GIS and secondary GIS are resolved; partial effectiveness is defined a } \\
\text { either primary GIS is improved; or primary GIS is resolved, but there were still unresolved secondary GIS. LOCF las } \\
\text { observation carried forward } \\
\text { b Exact } 95 \% \text { CI by Clopper and Pearson } \\
\text { c } p \text { value obtained from Exact test }\end{array}$} \\
\hline
\end{tabular}

second strategy, with eight of these patients (8/ $58 ; 14 \%$ of the total number of patients in this group) reporting complete or partial resolution with the combination of the two strategies (Fig. 2).

Of the patients who initiated on DE within 30 min of a meal, 26 experienced partial or no resolution on the initial strategy. Of these, 14 patients added the second strategy, with 12 of these patients $(12 / 59 ; 20 \%$ of the total number of patients in this group) reporting complete or partial resolution with the combination of the two strategies (Fig. 2).

Ultimately, at the end of treatment, 92 (78.6\%) patients (79 with complete effectiveness and 13 with partial effectiveness) experienced positive outcomes using either the initial, or the two combined, GIS management strategies: $47 / 58(81.0 \%)$ in the DE plus pantoprazole group (41 complete effectiveness plus six partial effectiveness) and 45/59 (76.3\%; NSD) in the DE after a meal group (39 complete effectiveness plus six partial effectiveness).

\section{Safety}

The two GIS management strategies were associated with similar AE profiles. Overall, $75.2 \%(88 / 117)$ of patients reported at least one AE. All but three of the AEs (bronchitis, dizziness, and headache) with an incidence of at least 5\% were GI events (Table 4). No statistically significant differences in AEs between the two GIS management groups were identified. Also of note, no new safety signals were detected in either treatment group.

Additional descriptive safety information is included under Supplemental Material.

\section{DISCUSSION}

The desire to find an alternative to warfarin has led to a series of clinical trials investigating the role of DOACs in the treatment of patients with NVAF, including direct thrombin inhibitors and factor Xa inhibitors [8]. Agents that specifically target a single step in the coagulation cascade 
Table 4 Adverse events with incidence at least 5\% by GIS management strategy, primary system organ class, and preferred term (treated set)

\begin{tabular}{llll}
\hline System organ class/preferred term & $\begin{array}{l}\text { DE after a meal } \\
(\boldsymbol{n}=59)\end{array}$ & $\begin{array}{l}\text { DE plus pantoprazole } \\
(\boldsymbol{n}=58)\end{array}$ & $\begin{array}{l}\text { Total } \\
(\boldsymbol{N}=\mathbf{1 1 7})\end{array}$ \\
\hline Total with AEs & $44(74.6)$ & $44(75.9)$ & $88(75.2)$ \\
Infections and infestations & $8(13.6)$ & $5(8.6)$ & $13(11.1)$ \\
Bronchitis & $3(5.1)$ & $1(1.7)$ & $4(3.4)$ \\
Nervous system disorders & $5(8.5)$ & $8(13.8)$ & $13(11.1)$ \\
Dizziness & $3(5.1)$ & $2(3.4)$ & $5(4.3)$ \\
Headache & $1(1.7)$ & $3(5.2)$ & $4(3.4)$ \\
GI disorders & $38(64.4)$ & $34(58.6)$ & $72(61.5)$ \\
Dyspepsia & $17(28.8)$ & $15(25.9)$ & $32(27.4)$ \\
Eructation & $13(22.0)$ & $10(17.2)$ & $23(19.7)$ \\
Abdominal discomfort & $7(11.9)$ & $13(22.4)$ & $20(17.1)$ \\
Abdominal distension & $8(13.6)$ & $12(20.7)$ & $20(17.1)$ \\
Epigastric discomfort & $9(15.3)$ & $10(17.2)$ & $19(16.2)$ \\
Abdominal pain upper & $8(13.6)$ & $10(17.2)$ & $18(15.4)$ \\
Nausea & $5(8.5)$ & $10(17.2)$ & $15(12.8)$ \\
Diarrhea & $7(11.9)$ & $7(12.1)$ & $14(12.0)$ \\
Regurgitation & $9(15.3)$ & $4(6.9)$ & $13(11.1)$ \\
Flatulence & $5(8.5)$ & $5(8.6)$ & $10(8.5)$ \\
Constipation & $3(5.1)$ & $4(6.9)$ & $7(6.0)$ \\
Defecation urgency & $4(6.8)$ & $2(3.4)$ & $6(5.1)$ \\
GI sounds abnormal & $4(6.8)$ & $2(3.4)$ & $6(5.1)$ \\
Feces hard & $1(1.7)$ & $3(5.2)$ & $4(3.4)$ \\
\hline Perentges & & &
\end{tabular}

Percentages are calculated using total number of patients per GIS management strategy as the denominator MedDRA version used for reporting: 17.0

Time to event for patients in the treated set is based on the day of randomization (assigned to a management strategy) $A E$ adverse event, $D E$ dabigatran etexilate, $G I$ gastrointestinal, GIS gastrointestinal symptom

offer substantial clinical advantages over warfarin, including predictable pharmacokinetics and pharmacodynamics, minimal medication and food interactions, and fixed dosing without the need for regular laboratory monitoring of coagulation status $[9,10]$. The first of the DOAC trials to conclude was the RE-LY trial, which compared two blinded doses of DE, a direct thrombin inhibitor, with warfarin (target international normalized ratio of 2.0-3.0) in an open-label fashion in 18,113 patients with NVAF [4]. The results of the RE-LY trial showed a statistically significant 35\% reduction in $\mathrm{RR}$ for stroke or systemic embolism with the 150-mg BID dose compared with warfarin in this patient 
population. Additionally, there was an approximately two-thirds reduction in the risk of intracranial bleeding, and an increased risk of major GI bleeding with the 150-mg dose, but similar risk with the 110-mg dose, resulting in an overall similar (DE 150-mg BID) or reduced (DE 110-mg BID) rate of major bleeding, versus warfarin [4]. Efficacy similar to that achieved with well-controlled warfarin was observed with the 110-mg BID dose of dabigatran. Although this dose is approved in Canada, it was not approved in the United States [11]. An alternate dose of 75-mg is approved in the United States for patients with a creatinine clearance of $15-<30 \mathrm{ml} / \mathrm{min}$.

In the RE-LY trial, the two DE dose regimens were generally well tolerated. However, dyspepsia-like GIS (which included upper abdominal pain, abdominal pain, abdominal discomfort, epigastric discomfort, and dyspepsia) was reported more often in patients receiving DE (110-mg: 11.8\%; 150-mg: 11.3\%) than in those receiving warfarin (5.8\%) [4]. A post hoc analysis of non-bleeding upper GI (NB-UGI) events in the RE-LY trial determined that these events generally fell into one of the four following groups (gastroesophageal reflux, upper abdominal pain and dyspepsia, dysmotility, or gastroduodenal injury) [6]. Of the patients on either dose of dabigatran, $4.0 \%$ discontinued treatment due to NB-UGI AEs (approximately half of these within the initial 3 months of therapy), compared with $1.7 \%$ of warfarin-treated controls (RR 2.34; 95\% CI 1.90-2.88\%; $p<0.001$ ) [6].

During the 6.7 years of follow-up to RE-LY in the RELY-ABLE trial, dyspepsia symptoms were reported in 602 (10.0\%) and 569 (9.4\%) patients receiving DE 110-mg and DE 150-mg, respectively [12]. As part of this long-term follow-up trial, the patients also completed a questionnaire designed to identify strategies employed to relieve their dyspepsia symptoms. Use of a proton pump inhibitor or taking dabigatran with meals were the strategies most commonly used, leading to symptomatic improvement in more than $85 \%$ of patients who reported these symptoms [12].

Given the magnitude of the reduction in stroke and embolic events in the RE-LY trial, a way to mitigate the GIS associated with DE would be a welcome development. Therefore, in the present clinical trial, two strategies for managing GIS were compared in patients with NVAF who were naïve to previous DE treatment. The first was the addition of the proton pump inhibitor pantoprazole 40-mg $\mathrm{OD}$, and the second was the strategy of taking DE within $30 \mathrm{~min}$ after a meal. The trial employed an "add on" design in which patients who did not respond to the initial randomized strategy after 4 weeks added the alternative strategy for a further 4 weeks. A total of 117 (11.0\%) patients reported GIS, a rate that was remarkably similar to the dyspepsia-like GIS rates reported in the RE-LY trial (110-mg: 11.8\%; 150-mg: 11.3\%) [4].

Based on the incidence of GIS among patients receiving DE in the RE-LY trial, it was estimated that $6-12 \%$ of patients would report GIS in this study. Therefore, with a planned sample size of 1200 patients, an estimated 144 patients were expected to develop GIS. As a consequence of the slower than expected recruitment, once 117 patients with GIS were identified, ongoing recruitment terminated and the study was completed with the patients already enrolled.

In the present trial, the two GIS management strategies provided high rates of success, either alone or in combination. As many as $61.5 \%$ of patients responded to their initial management strategy with "complete resolution" of symptoms. At the 4-week assessment, the 
dabigatran plus pantoprazole strategy was more effective in terms of partial or complete resolution of GIS than the dabigatran within 30 min of a meal strategy (86.2\% versus $67.8 \%$, respectively; $p=0.0273$ ).

At the end of the trial, of the 59 patients initially randomized to the DE after a meal strategy, 45 (76.3\%) patients in this group had partial or complete resolution with either the single or combined management strategies. In the 58 patients initially randomized to the $\mathrm{DE}$ plus pantoprazole strategy, 47 (81.0\%) in this group had partial or complete resolution with the initial or combined management strategies. Not surprisingly, the two strategies in combination led to very similar rates of complete or partial resolution of the patient's GIS. At the end of the trial, only 25 (21.4\%) patients with GIS failed to respond to either a single strategy, or a combination of the two GIS management strategies.

The underlying cause(s) of DE-related GI AEs is not understood [6]. To enhance DE absorption, the DE-coated capsules have a tartaric acid core that promotes a low $\mathrm{pH}$ at the site of absorption [13]. It has been proposed that this acidity may lead to an increase in GIS. However, other medications have been developed with similar acid core formulations [14], and they have not been associated with reported increases in GIS. In the RE-LY trial, $14 \%$ of the patients in both the DE and warfarin treatment groups were taking a proton pump inhibitor at baseline [4].

In clinical trials, patients are encouraged to stay on medication even if they experience mild to moderate AEs. However, in clinical practice, patients are routinely and rapidly switched to another drug. The present evidence that simple management strategies can prevent GIS in patients treated with DE may enable physicians to utilize DE as first-line therapy in the approved indications: reduction in risk of stroke and systemic embolism in NVAF, treatment of DVT and PE, reduction in risk of DVT and PE in patients who have been previously treated, and the prophylaxis of DVT and $\mathrm{PE}$ in patients who have undergone hip or knee replacement surgery $[2,3]$.

A number of limitations associated with the present trial should be noted, including the non-specific nature of the symptoms reported, and the reliance on patient self-reporting of their symptoms which may each have contributed some bias to the reported outcomes. However, to improve consistency in self-reporting of GIS, patient responses were recorded using the validated GSRS questionnaire [15]. Also, the GIS trial did not evaluate patients who experienced prolonged exposure to DE. However, it was noted previously in the RE-LY trial that GIS generally occurred early (primarily in the first 3 months) in a course of DE therapy [6]. Another limitation of this study was the absence of a non-GIS management, untreated control group, since the natural history of non-drug related dyspepsia and associated GI disorders is complex and highly variable [16]. This was due in part to the relatively small sample size of patients who were randomized.

\section{CONCLUSION}

In conclusion, dabigatran was generally well tolerated by the patient population in this study. Approximately $11 \%$ of patients enrolled in the study reported GIS during the 3-month treatment period and were randomized to a management strategy (117/1067). Of the 117 patients randomized, approximately 79\% (92/ 117) experienced complete or partial relief of symptoms with one or both of the management 
strategies. Overall, the majority of patients enrolled either did not experience GIS, or had symptoms that resolved.

\section{ACKNOWLEDGMENTS}

This work was supported by Boehringer Ingelheim Pharmaceuticals, Inc. (BIPI). Writing, editorial support, and/or formatting assistance was provided by José L. Walewski, Ph.D., of Envision Scientific Solutions, which was contracted and compensated by BIPI for these services. The authors received no compensation related to the development of the manuscript. The authors would like to acknowledge the contributions of Dr. Carol Duffy during the late stages of this clinical trial, and Dr. Janet Schnee for her contributions to this trial and her review of early versions of this manuscript. All authors had full access to all of the data in this study and take complete responsibility for the integrity of the data and accuracy of the data analysis. The authors meet criteria for authorship as recommended by the International Committee of Medical Journal Editors (ICMJE) and were fully responsible for all content and editorial decisions, and were involved at all stages of manuscript development.

Disclosures. Daniel O'Dea was the Principal Investigator of the GIS trial, which was funded by Boehringer Ingelheim Pharmaceuticals, Inc. (BIPI). Jacqueline Whetteckey is an employee of BIPI, the manufacturer of dabigatran etexilate, and the funding source for this clinical trial. Naitee Ting is an employee of BIPI, the manufacturer of dabigatran etexilate, and the funding source for this clinical trial.
Open Access. This article is distributed under the terms of the Creative Commons Attribution-NonCommercial 4.0 International License (http://creativecommons.org/licenses/ by-nc/4.0/), which permits any noncommercial use, distribution, and reproduction in any medium, provided you give appropriate credit to the original author(s) and the source, provide a link to the Creative Commons license, and indicate if changes were made.

\section{REFERENCES}

1. Stangier J, Clemens A. Pharmacology, pharmacokinetics, and pharmacodynamics of dabigatran etexilate, an oral direct thrombin inhibitor. Clin Appl Thromb Hemost. 2009;15(Suppl 1):9S-16S.

2. Boehringer Ingelheim Pharmaceuticals, Inc. Pradaxa prescribing information. November, 2015. http://bit.ly/1r26yMg. Accessed 22 Dec 2015.

3. Boehringer Ingelheim Canada Ltd. Pradaxa product monograph. Boehringer Ingelheim Canada Ltd. 2016. http://www.boehringer-ingelheim.ca/ content/dam/internet/opu/ca_EN/documents/human health/product_monograph/PradaxaPMEN.pdf. Accessed Apr 112016.

4. Connolly SJ, Ezekowitz MD, Yusuf S, Eikelboom J, Oldgren J, Parekh A, et al. Dabigatran versus warfarin in patients with atrial fibrillation. N Engl J Med. 2009;361(12):1139-51.

5. Connolly SJ, Ezekowitz MD, Yusuf S, Reilly PA, Wallentin L. Randomized Evaluation of Long-Term Anticoagulation Therapy Investigators. Newly identified events in the RE-LY trial. N Engl J Med. 2010;363(19):1875-6.

6. Bytzer P, Connolly SJ, Yang S, Ezekowitz M, Formella S, Reilly PA, et al. Analysis of upper gastrointestinal adverse events among patients given dabigatran in the RE-LY trial. Clin Gastroenterol Hepatol. 2013;11(3):246-52.e1-5.

7. Nieuwlaat R, Healey JS, Ezekowitz M, Reilly P, Formella S, Wallentin L, et al. Management of dyspepsia symptoms on dabigatran during RELY-ABLE: long-term follow-up study after RE-LY. European Society of Cardiology (ESC) Congress 2013; 31 Aug-4 Sep 2013; Amsterdam, 
The Netherlands. http://bit.ly/1Oir7fi. Accessed 15 Aug 2015.

8. Halperin JL, Dorian P. Trials of novel oral anticoagulants for stroke prevention in patients with non-valvular atrial fibrillation. Curr Cardiol Rev. 2014;10(4):297-302.

9. Greinacher A, Thiele T, Selleng K. Reversal of anticoagulants: an overview of current developments. Thromb Haemost. 2015;113(5):931-42.

10. Siegal DM, Crowther MA. Acute management of bleeding in patients on novel oral anticoagulants. Eur Heart J. 2013;34(7):489-98b.

11. Beasley BN, Unger EF, Temple R. Anticoagulant options-why the FDA approved a higher but not a lower dose of dabigatran. $\mathrm{N}$ Engl $\mathrm{J}$ Med. 2011;364(19):1788-90.

12. Ezekowitz MD, Eikelboom J, Oldgren J, Reilly PA, Brueckmann $\mathrm{M}$, Kent $\mathrm{AP}$, et al. Long-term evaluation of dabigatran 150 vs. $110 \mathrm{mg}$ twice a day in patients with non-valvular atrial fibrillation. Europace. 2016;18(7):973-8.

13. Eisert WG, Hauel N, Stangier J, Wienen W, Clemens A, van Ryn J. Dabigatran: an oral novel potent reversible nonpeptide inhibitor of thrombin. Arterioscler Thromb Vasc Biol. 2010;30(10):1885-9.

14. Pharmatrans Sanaq AG Pharmaceuticals. TAP ${ }^{\circledR}$ Tartaric acid pellet. 2012. http://bit.ly/1O6P3GY. Accessed 2 Jun 2015.

15. Kulich KR, Madisch A, Pacini F, Pique JM, Regula J, Van Rensburg CJ, et al. Reliability and validity of the Gastrointestinal Symptom Rating Scale (GSRS) and Quality of Life in Reflux and Dyspepsia (QOLRAD) questionnaire in dyspepsia: a six-country study. Health Qual Life Outcomes. 2008;6:12.

16. Olafsdottir LB, Gudjonsson $\mathrm{H}$, Jonsdottir $\mathrm{HH}$, Bjornsson E, Thjodleifsson B. Natural history of functional gastrointestinal disorders: comparison of two longitudinal population-based studies. Dig Liver Dis. 2012;44(3):211-7. 